\title{
Integrating Community Health Workers into Safety-Net Primary Care for Diabetes Prevention: Qualitative Analysis of Clinicians' Perspectives
}

\author{
Radhika Gore, $P h D^{7}{ }^{\infty}$, Ariel Brown, $B A^{2}$, Garseng Wong, $B A^{7}$, \\ Scott Sherman, MD, MPH ${ }^{1,3}$, Mark Schwartz, MD ${ }^{1,3}$, and Nadia Islam, $P h D^{7}$
}

${ }^{1}$ NYU School of Medicine, New York, NY, USA; ${ }^{2}$ Sidney Kimmel Medical College, Philadelphia, PA, USA; ${ }^{3}$ VA NY Harbor Health Care System, New York, NY, USA

BACKGROUND: Evidence shows community health workers (CHWs) can effectively deliver proven behaviorchange strategies to prevent type 2 diabetes mellitus (diabetes) and enhance preventive care efforts in primary care for minority and low-income populations. However, operational details to integrate CHWs into primary care practice remain less well known.

OBJECTIVE: To examine clinicians' perceptions about working with $\mathrm{CHWs}$ for diabetes prevention in safety-net primary care.

SETTING: Clinicians are primary care physicians and nurses at two New York City safety-net hospitals participating in CHORD (Community Health Outreach to Reduce Diabetes). CHORD is a cluster-randomized trial testing a $\mathrm{CHW}$ intervention to prevent diabetes.

DESIGN: Guided by the Consolidated Framework for Implementation Research, we studied how features of the CHW model and organizational context of the primary care practices influenced clinicians' perspectives about the acceptability, appropriateness, and feasibility of a diabetes-prevention CHW program. Data were collected pre-intervention using semi-structured interviews $(n=$ 18 ) and a 20-item survey $(n=54)$.

APPROACH: Both survey and interview questions covered clinicians' perspectives on diabetes prevention, attitudes and beliefs about CHWs' role, expectations in working with CHWs, and use of clinic- and community-based diabetes- prevention resources. Survey responses were descriptively analyzed. Interviews were coded using a mix of deductive and inductive approaches for thematic analysis.

KEY RESULTS: Eighty-seven percent of survey respondents agreed CHWs could help in preventing diabetes; $83 \%$ reported interest in working with CHWs. Ninety-one percent were aware of clinic-based prevention resources; only $11 \%$ were aware of community resources. Clinicians supported CHWs' cultural competency and neighborhood reach, but expressed concerns about the adequacy of

Prior Presentations Preliminary findings from this research were pre sented at the Society for General Internal Medicine Annual Meeting, April 11-14, 2018, Denver, CO, and the 11th Annual Conference on the Science of Dissemination and Implementation in Health, December 3-5, 2018, Washington, DC

Received May 17, 2019

Revised October 7, 2019

Accepted November 25, 2019

Published online December 17, 2019
CHWs' training; public and professional emphasis on diabetes treatment over prevention; and added workload and communication with CHWs.

CONCLUSIONS: Clinicians found CHWs appropriate for diabetes prevention in safety-net settings. However, disseminating high-quality evidence about CHWs' effectiveness and operations is needed to overcome concerns about integrating $\mathrm{CHWs}$ in primary care.

KEY WORDS: community health worker; diabetes prevention; implementation research; primary care; health disparities.

J Gen Intern Med 35(4):1199-210

DOI: $10.1007 / \mathrm{s} 11606-019-05581-8$

(c) Society of General Internal Medicine 2019

\section{INTRODUCTION}

Type 2 diabetes mellitus (diabetes) is a preventable chronic disease that affects $9.4 \%$ of US adults. Another 84 million American adults have prediabetes, with glycated hemoglobin (A1c) in the range 5.7\% through $6.4 \%$, just below the level for diabetes. ${ }^{1}$ Half of people with prediabetes may develop diabetes over the next decade, but the progression can be delayed or prevented. ${ }^{2}$ Notably, primary prevention - preventing the occurrence of diabetes - can be achieved and maintained in the long term through proven behavior-change interventions focusing on weight loss and physical activity. ${ }^{2-4}$ In contrast, secondary prevention (i.e., preventing complications among those who have diabetes) and treatment of diabetes may involve more than lifestyle modifications, such as managing blood glucose levels through oral medications and insulin therapy. However, primary care (PC) teams infrequently refer patients to evidence-based primary prevention programs. ${ }^{5}$ Barriers to diabetes prevention include insufficient time, skills, and tools for clinicians to conduct behavioral counseling interventions and unclear delegation of non-visit-based outreach tasks. ${ }^{6,7}$

One strategy that may overcome these barriers is the addition of community health workers (CHWs) to health care teams. CHWs are frontline public health workers who are trusted members of or closely familiar with the community they serve.$^{8-11} \mathrm{CHWs}$ with appropriate training can serve as 
effective peer coaches by providing culturally congruent counseling to promote healthy behaviors and motivate patients to engage in proven disease prevention strategies. ${ }^{12-17} \mathrm{CHWs}$ integrated into PC teams can improve care for patients with chronic disease at modest cost, improve preventive care, and address social determinants of health. ${ }^{9,}{ }^{18-22}$

Despite CHWs' potential to enhance diabetes prevention efforts, there is limited evidence about implementation processes to integrate CHWs into PC teams. ${ }^{20,23-25}$ Studies show $\mathrm{PC}$ team members may not understand the range of CHWs' duties, such as CHWs' capacity to conduct communityresource navigation and not only provide health education, ${ }^{26}$ and may lack familiarity with CHW program details, such as optimal frequency of case conferences, CHW caseload, and procedures for referring patients to CHWs. ${ }^{24,}{ }^{26-28}$ Moreover, studies of CHW integration in PC often lack a conceptual frame to connect contextual factors (e.g., organizational setting) and implementation outcomes (e.g., stakeholder perspectives on program acceptability, appropriateness, and feasibility), and miss opportunities to advance health equity research and develop disparity-reducing implementation strategies. ${ }^{29}$

Therefore, we applied the Consolidated Framework for Implementation Research (CFIR) to thematically analyze how contextual factors related to the intervention, organization, and external environment influence the possibility of integrating CHWs into PC teams in safety-net settings. We present findings from an implementation study of the Community Health Outreach to Reduce Diabetes (CHORD) trial, conducted prior to intervention launch. CHORD, described in detail elsewhere, ${ }^{30}$ is a cluster-randomized pragmatic trial to test the effectiveness of CHW-led primary prevention of diabetes in safety-net settings through encouraging lifestyle change, offering social support, and referring prediabetes patients to community-based diabetes prevention resources. Our aim in this end-user inquiry was to examine clinicians' perceptions about CHWs to inform program design, with the larger objective to develop a thematic scheme of contextual factors likely to influence $\mathrm{CHW}$ integration in primary care.

\section{METHODS}

\section{Research Setting}

The CHORD trial is being implemented at $\mathrm{PC}$ practices at the Manhattan campus of the VA NY Harbor Healthcare System and Bellevue Hospital Center, the flagship of New York City's municipal hospital system. ${ }^{30}$ The prevalence rate of diabetes among adult PC patients is an estimated $20 \%$ at the VA and $15 \%$ at Bellevue Hospital, compared with $10.5 \%$ in New York City. ${ }^{31,32}$ At both sites, a substantial proportion of patients are at risk for diabetes, are low-income, and belong to racial/ethnic minority groups. Eligibility criteria for patients in CHORD are as follows: aged 18-75 years, enrolled in a PC clinic, no diabetes diagnosis at baseline (based on ICD codes and A1c results), and at least one A1c result in the prediabetes range $(5.7-6.4 \%)$ in the 5 years prior to the intervention. ${ }^{30}$

While PC teams at both sites are multi-professional- patients have access to mental health professionals, social workers, pharmacists, and nutritionists attached to teams (Table 1) - teams do not work with CHWs for diabetes prevention. This makes the sites an ideal context in which to explore clinicians' a priori perspectives on working with $\mathrm{CHWs}$ to reach diverse vulnerable populations.

\section{Conceptual Framework and Research Approach}

We use a case study approach, which is relevant to analyze links between the operational and contextual features of a case. ${ }^{33}$ In our study, the case is an intervention, namely CHW-led diabetes prevention. The safety-net PC sites constitute its organizational and locational bounds, and the CHORD study's duration

Table 1 Description of Primary Care Teams and Data Collection at the Manhattan VA and Bellevue Hospital

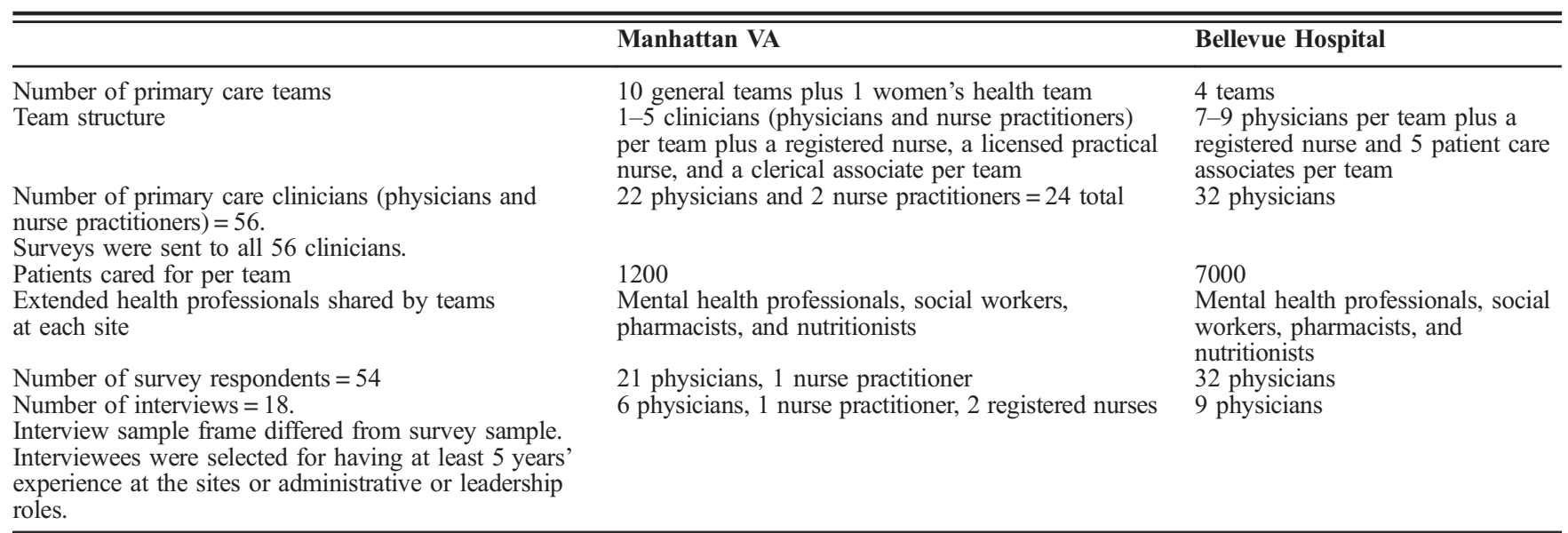


(2018-2021) constitutes its temporal bound, though here we focus on pre-implementation results. ${ }^{34}$ In this instrumental case study, the issue we explore is the relationship between context and implementation outcomes. ${ }^{34}$ Contextual factors can affect the translation of interventions across PC settings. ${ }^{35}$ To systematically relate internal and external contexts to potential implementation outcomes, we draw on the CFIR. ${ }^{36}$ We focus on three relevant CFIR domains - characteristics of the intervention, inner setting of the organization, and outer setting of the organization - to examine how context shapes PC team members' perceptions about CHWs prior to CHORD implementation. The two remaining CFIR domains - process of implementation and characteristics of individuals involved in implementation - are not applicable in this baseline (preimplementation) study of clinicians' perspectives.

We examine clinicians' perspectives on three implementation outcomes: whether team members find CHWs to be acceptable, appropriate, and feasible to integrate in their practice. ${ }^{37}$ (Other implementation outcomes, such as adoption, cost, fidelity, penetration, and sustainability, are of greater relevance once implementation is underway.) We define $a c$ ceptability as stakeholders' perception that the intervention is agreeable or satisfactory in principle, based on their knowledge of or direct experience with its elements. Appropriateness is the perceived fit or relevance of the intervention for a particular population and practice setting. Feasibility is the extent to which an innovation can be successfully used or carried out within a given setting.

\section{Data Collection and Main Variables}

Data were collected using a self-administered survey to assess clinicians' attitudes and practices along specified domains and semi-structured in-person interviews to further explore their views through open-ended dialogue.

\section{Survey}

A 20-item questionnaire was administered to all 56 clinicians at the two sites, of whom 54 responded (Table 1). Survey questions queried four domains: self-efficacy about preventing diabetes among at-risk patients, beliefs about CHWs' role and effectiveness, interest in working with CHWs, and use of diabetes prevention resources. The items captured aspects of clinicians' attitudes and perspectives that CHWs could potentially impact over time. Responses used a 1-5 scale, from strongly disagree to strongly agree. Thirty-eight clinicians were surveyed in-person. Reminder emails were sent to non-responders with a link to an online questionnaire; 16 responded online. Senior research team members additionally encouraged survey participation, helping to achieve a high response rate.

\section{Interviews}

In-person interviews, lasting 15-30 min, were conducted with 18 PC team members, 9 at each site (Table 1), purposively selected based on having at least 5 years of work experience and leadership or influence within the practice. Informed consent was obtained for participation in the interviews and for audio-recording of interviews. Interviews were conducted by three research team members using guides containing questions covering usual practices in panel management and diabetes prevention practices, use of clinic- and communitybased diabetes prevention resources, beliefs about CHWs' role, and expectations and concerns about working with CHWs for diabetes prevention (see Annexure). Interviews focused not on CHORD details, which were being finalized as we conducted the interviews, but on broad topics investigating clinicians' perspectives about CHWs in general.

All research procedures were approved by the NYU School of Medicine Institutional Review Board, the VA New York Harbor Healthcare System Office of Research \& Development Institutional Review Board, and the Bellevue Research Review Committee and New York City Health + Hospital's Research Administration.

\section{Data Analysis}

Questionnaire items were summarized as the percentage of subjects that agreed or strongly agreed (responses of 4 or 5 ) for each item. Our small sample size limits factor analysis. We therefore provide results for individual items rather than treat the clusters as scales, and present the heterogeneity among items within each cluster.

To analyze the interviews, we used a mix of deductive and inductive approaches for thematic analysis. Transcribed interviews were analyzed in two stages, using the Atlas.ti software. The first focused on implementation outcomes: transcripts were coded for informants' perspectives on acceptability, appropriateness, and feasibility of working with CHWs at their practice. In the second stage, coded transcripts were re-read to inductively identify themes reflecting contextual factors, which we interpreted in relation to CFIR constructs and subconstructs. That is, we first inductively looked for themes that reflected contextual factors. We generated descriptive codes for these emergent themes. We then examined these themes to see if and how they corresponded to CFIR constructs or indicated connections between CFIR constructs. Three research team members independently coded all interviews, then met to compare themes and resolve any discrepancies in interpretation of responses.

Although we did not run a member check, we discussed findings with our clinical champions at each site and among all research team members, two of whom are physicians at one of the sites, which helped to refine and confirm the analysis.

\section{Researcher Characteristics}

The analysis is informed by the research team's collective knowledge of the PC practices at the two study sites. Six team members were involved in the data collection and analysis. Two team members are physicians; both work at one of the study sites. Two members (non-physicians) hold $\mathrm{PhDs}$ with 
experience conducting qualitative research in public health. Three members (the two physicians plus one $\mathrm{PhD}$ ) were involved in research design and data analysis but not data collection. The remaining three members were uninvolved in program implementation, and collected, transcribed, and analyzed data.

\section{FINDINGS}

\section{Survey Results}

Survey results characterize physician demographics and their knowledge and awareness of CHWs and diabetes prevention resources (Table 2). Survey results show clinicians' high interest in working with CHWs as well as their low knowledge and use of community resources for healthy behavior change. Alpha coefficients for three clusters are strong $(>0.8)$. Yet clinicians' self-efficacy is variable and low in some areas: helping patients lose weight, change physical activity, and prevent diabetes (items 3,5, and 6). In the fourth cluster, clinicians report low awareness of and readiness to refer patients to community resources (items 17,18 , and 20), which is where CHWs can be particularly helpful.

\section{Contextual Factors Underlying Clinicians' Perceptions}

Qualitative study findings are organized below by CFIR constructs and subconstructs. Table 3 lists and defines the relevant
CFIR subconstructs. Table 4 summarizes the findings and provides additional representative quotes in support of the analysis.

\section{Intervention Characteristics}

Clinicians' acceptance of CHWs reflected the nature of evidence supporting their belief that an intervention will have desired outcomes. Clinicians cited mostly anecdotal sources of evidence about CHWs, such as their patients' and, in a few cases, their own experiences. Clinicians appeared to find these experiences positive and valid: they discussed how CHWs could play a valuable role in bringing culturally and linguistically concordant peer-coaching to patients in their communities. They reported that patients who had worked with CHWs had been enthusiastic about the CHW "also speak[ing] Spanish," or that the CHW was, like the patient, also Bengali and showed the patient "how to work with the rice issue." One physician noted that in his experience, it was powerful for a veteran to listen to another veteran recount the health treatment rather than to hear it from the doctor.

Clinicians additionally recounted the relative advantages that CHWs offered over solely clinic-based care. One physician noted that diabetes prevention was appropriately addressed outside the clinic. CHWs could reinforce a clinicians' message "carry[ing] on conversations that start in the clinical setting and extend into the community." CHWs could help "to get healthcare out of the hospital," said another physician-they

Table 2 Survey Items and Subscales with Their Cronbach's Alpha Coefficient and Percent That Agree or Strongly Agree

\begin{tabular}{llll}
\hline \hline Subscale & Alpha & Survey item & \% agree or strongly
\end{tabular}
agree

Preventing diabetes

Beliefs about community health workers (CHWs)

Working with community health workers (CHWs)

Use of diabetes prevention resources

0.81

1. I can ider diabetes

2. I am familiar with the evidence base on effective strategies for diabetes prevention.

3. I can help my overweight or obese patients successfully lose weight.

4. I can help my patients at risk for diabetes adhere to dietary modifications.

5. I can help my patients at risk for diabetes adhere to changes in their physical activity.

6. I can prevent diabetes among my patients at risk of diabetes.

0.92 7. CHWs can reinforce my counseling of patients to prevent diabetes.

8. CHWs can be effective at helping my patients to improve their diet.

9. CHWs can be effective at helping patients to improve their exercise habits.

10. CHWs are able to motivate patients at risk of diabetes to enroll in prevention programs.

0.93 11. I am interested to work with CHWs to prevent diabetes among my highrisk patients.

12. I believe that adding CHWs to my team will improve diabetes prevention among my high-risk patients.

13. I will be able to work with CHWs to motivate patients to improve their diet.

14. I will be able to work with CHWs to motivate my patients to improve their exercise habits.

15. I will be able to work with CHWs to engage my patients in diabetes prevention programs.

0.55 16. I am aware of diabetes prevention resources in the clinic.

17. I am aware of diabetes prevention resources in the community.

18. I follow up with my patients after linking them to diabetes prevention resources.

19. I routinely refer patients to diabetes prevention resources in the clinic. 20 . I routinely refer patients to diabetes prevention resources in the community.
$100.0 \%$

$88.9 \%$

$44.4 \%$

$50.0 \%$

$44.4 \%$

$44.4 \%$

$92.6 \%$

$90.7 \%$

$81.5 \%$

$83.3 \%$

$92.6 \%$

$83.3 \%$

$79.6 \%$

$79.6 \%$

$81.5 \%$

$90.7 \%$

$11.1 \%$

$48.1 \%$

$53.7 \%$

$1.9 \%$ 
Table 3 Definitions of CFIR Subconstructs

\begin{tabular}{|c|c|c|}
\hline $\begin{array}{l}\text { CFIR construct and } \\
\text { subconstruct }\end{array}$ & Definition* & Themes from data reflecting CFIR constructs \\
\hline \multicolumn{3}{|c|}{ Characteristics of the intervention } \\
\hline $\begin{array}{l}\text { Evidence strength and } \\
\text { quality }\end{array}$ & $\begin{array}{l}\text { Stakeholders' perceptions about the quality and validity of } \\
\text { evidence supporting their belief that the intervention will } \\
\text { have desired outcomes. Sources of evidence can include } \\
\text { published literature and patients', peers', and personal } \\
\text { anecdotal reports. }\end{array}$ & $\begin{array}{l}\text { Clinicians knew enough about CHWs to find them broadly } \\
\text { acceptable and appropriate for diabetes prevention in their } \\
\text { clinic setting, but the evidence they had did not allay their } \\
\text { concerns about whether CHWs will fully benefit patients } \\
\text { and about operationalizing CHW programs in PC practice. }\end{array}$ \\
\hline Relative advantage & $\begin{array}{l}\text { Stakeholders' perception of the advantage of implementing } \\
\text { the intervention vs. alternative solutions. }\end{array}$ & \\
\hline \multicolumn{3}{|c|}{ tie minervention vs. allemauve somutions. } \\
\hline $\begin{array}{l}\text { Patient needs and } \\
\text { resources }\end{array}$ & $\begin{array}{l}\text { The extent to which patients' needs and constraints to } \\
\text { accessing care and services, which are factors external to the } \\
\text { organization, are accurately known and prioritized by the } \\
\text { organization. }\end{array}$ & $\begin{array}{l}\text { Clinicians perceived CHWs as appropriate for their } \\
\text { patients, yet were concerned that diabetes treatment rather } \\
\text { than prevention was the more urgent priority. } \\
\text { Clinicians' suggestions for ways to feasibly integrate }\end{array}$ \\
\hline $\begin{array}{l}\text { External policies and } \\
\text { incentives }\end{array}$ & $\begin{array}{l}\text { Includes policy and regulations (governmental or other } \\
\text { central entity), external mandates, guidelines and } \\
\text { recommendations, pay-for-performance incentives, and pub- } \\
\text { lic reporting. }\end{array}$ & $\begin{array}{l}\text { CHWs into their practices underscored their concerns about } \\
\text { the relative importance of prevention vs. treatment, } \\
\text { reflecting external debates and professional views on } \\
\text { resource allocation for prevention vs. treatment. }\end{array}$ \\
\hline \multicolumn{3}{|r|}{ 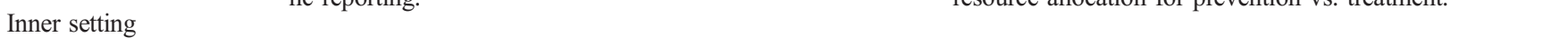 } \\
\hline $\begin{array}{l}\text { Implementation } \\
\text { climate }\end{array}$ & $\begin{array}{l}\text { Includes the organization's absorptive capacity for change; } \\
\text { compatibility between organizational incentives and priorities } \\
\text { and those addressed by the intervention; and involved } \\
\text { individuals' shared receptivity to the intervention and } \\
\text { readiness to learn. }\end{array}$ & $\begin{array}{l}\text { Clinicians were open to innovation and perceived CHWs as } \\
\text { appropriate for their practices. However, clinicians were } \\
\text { concerned that diabetes treatment rather than prevention } \\
\text { was the more urgent priority. } \\
\text { Clinicians' suggestions for ways to feasibly integrate }\end{array}$ \\
\hline $\begin{array}{l}\text { Networks and } \\
\text { communications } \\
\text { Structural } \\
\text { characteristics }\end{array}$ & $\begin{array}{l}\text { The nature and quality of formal and informal networks, } \\
\text { relationships, and communication in the organization. } \\
\text { The social architecture of an organization, including how } \\
\text { people are clustered into groups or teams, how labor and } \\
\text { functional skills are differentiated across groups, and how } \\
\text { groups are coordinated. }\end{array}$ & $\begin{array}{l}\text { CHWs into their practices underscored their concerns about } \\
\text { the logistics, modes, and content of information exchange } \\
\text { between providers and CHWs, as well as aligning CHWs' } \\
\text { priorities, roles, and workflow with those of the rest of the } \\
\text { PC team. }\end{array}$ \\
\hline
\end{tabular}

*All definitions are adapted from Damschroder et al. ${ }^{36}$

could be "in people's neighborhoods and in people's houses and in people's communities."

Clinicians' views of CHWs' appropriateness were further shaped by their perception that diabetes prevention was typically accorded lower priority than treatment and that new approaches were needed for preventive care. In this regard, CHWs offered a relative advantage over existing programs. A physician emphasized the need to "think out of the box at this point," and that for a significant number of people, "the traditional approach" - typically requiring patients to autonomously stay engaged with diet and exercise programs - was "not working."

Yet clinicians expressed uncertainty (suggesting low access to evidence) about how CHWs would achieve desired effects and what unintended consequences might follow. Clinicians did not cite knowledge or evidence of adverse effects of CHWs; our findings suggest clinicians had limited exposure to CHWs and CHW-related evidence, which may have contributed to their uncertainty about CHW implementation. Physicians were concerned, for example, about CHWs' background, training, and skills to deliver health messages accurately, to convey "the right balance of risk and benefit." They questioned whether CHWs' role would be defined so that they could balance "their autonomy and their ability to do things independently," without their lack of formal training posing a risk to the patient. CHWs might become "a very blunt object" to help with prediabetes if they did not understand how "patients' lives are extremely hard and that losing weight or getting more exercise in their day just might not be a priority," said one physician.

\section{Outer Setting}

Clinicians' perspectives on CHWs' appropriateness reflected their awareness of patient needs and resources. A physician expressed that getting people to change their diets was not only about a "[health] knowledge deficit," it also required overcoming people's closely held beliefs, which made it hard for them to absorb scientific information. One physician noted that she rarely talked to patients about resources in the patients' neighborhood, that she did not know what those were, and hence a CHW program that "look[ed] at the community ... would be really worthwhile." Clinicians thought that cultural and social barriers to healthy behavior had to be tackled from within the community.

Simultaneously, they noted that the patient profile at safety-net organizations implied a significant prevalence of diabetes and created an expectation to prioritize diabetes treatment over prevention. It showed how external policies and incentives, including regulations, mandates, recommendations, and public reporting, might influence clinicians' perspectives on CHWs. As one physician put it, "people [meaning other health system actors] will wonder why are we assigning community health workers to patients with prediabetes when I have hundreds of patients 
Table 4 Influence of Context on Implementation Outcomes

\begin{tabular}{|c|c|c|c|}
\hline $\begin{array}{l}\text { CFIR construct and } \\
\text { subconstruct }\end{array}$ & Clinicians' perceptions about CHWs & Representative quotes & $\begin{array}{l}\text { Influence of context on } \\
\text { implementation outcome }\end{array}$ \\
\hline
\end{tabular}

\section{subconstruct}

Characteristics of the

intervention:

Evidence strength

Characteristics of the intervention:

Evidence strength

Characteristics of the intervention:

Relative advantage

Characteristics of the intervention:

Relative advantage

Outer setting: Patient needs and resources
Clinicians cited their patients' and, in a few cases, their own experiences, discussing how CHWs could play a valuable role in bringing culturally and linguistically concordant and personalized peer-coaching to patients in their communities.

Clinicians expressed several concerns about CHWs: whether CHWs would have the background and training to deliver health messages accurately and convey the right balance of risk and benefit to the patient; whether they would be able to independently work with patients without their lack of formal training posing a risk to patients; whether they would recognize that patients had difficult lives; and whether they would appropriately target those patients who needed support.

Clinicians felt CHWs offered an advantage over clinic-based care because preventing diabetes entailed patients' actions at home. CHWs could reinforce a physician's message outside the clinic, could be in people's neighborhoods and homes.

Clinicians said that CHWs offered an advantage over existing programs, which were focused on diabetes treatment and not prevention, or which had seen flagging interest from patients New approaches were needed for preventive care.

According to clinicians, features of the patient population made CHWs an appropriate intervention for lowincome, racial/ethnic minority populations because clinicians knew little about and rarely talked to patients about resources in patients' neighborhood. Getting people to change their diets and lifestyles entailed overcoming closely held beliefs and cultural and social barriers to healthy behavior in the community, requiring action from within the community.
Several physicians said they had not personally worked with CHWs: "I've heard the term, but I really do not know any details, and I certainly do not have any experience with them," said one physician. But they also commonly understood CHWs as "people from the community that are trained to try to address some of the ... therapeutic lifestyle changes for members of their community or people of the community that they are from," as one physician expressed. CHWs could "make targeted interventions that would be appropriate for that person as opposed to just saying "do not eat so much rice", said a physician.

A physician wondered whether CHWs would be: "[Trained] in the concept of behavioral change, and not just motivational interviewing. It's really about understanding how hard this is for patients to change and to be sympathetic and understanding and not feeling like they have to show a change and pressure people."

One physician worried that

"outsourcing" behavioral counseling to somebody else might break his bond with the patient.

Preventing diabetes "happens not from a physician's intervention but maybe with a physician's counseling or advice,... it happens with a patient on their own at home making their own decisions in real time." CHWs could "go to their house and see what's literally ... what's in those cabinets," said a physician.

A physician said that a CHW could be "another point of contact, somebody who could "reach out and say hey, how's it going, what's going on, to just keep pushing a message."

Physicians only saw a patient

face-to-face from time to time, while

CHWs could "get out to patients and spend actual time with them," said a registered nurse.

A nurse practitioner noted that some patients wanted neither to stay nor return another day for an appointment with a nutritionist, that they did not think that "nutrition is something that's worthwhile to come for an appointment ... Lifestyle changes are hard to make, especially ones that are around eating." "If there's somebody that comes from Bed-Stuy and knows what's around in that neighborhood, they'd be much more effective in interacting with our patients [from that neighborhood]," said a physician.

Engendering healthy behavior change among their patients could not be solved simply through additional staff or resources, because the greatest obstacle was "activating the patient," that is, "getting the patient to the right place - right place as in right physical place, right mental place, to get the
Clinicians' anecdotal and experiential views about CHWs' cultural competence supported perceived acceptability and appropriateness of CHWs.

Clinicians' lack of first-hand experience with CHWs and apparently low exposure to programmatic and evaluative evidence about CHW models created ambivalence about CHWs' effects and unintended consequences, and thus diminished perceived acceptability and appropriateness.

Lifestyle and behavior-change component of diabetes prevention and CHWs' capacity to reach patients at home or in the community supported perceived appropriateness of CHWs.

Clinicians' perception that patients would respond to new initiatives supported perceived appropriateness.

Clinicians recognized that CHWs' familiarity with communities and neighborhoods enabled them to reach their low-income, minority patients, which supported perceived appropriateness. 
Table 4. (continued)

\begin{tabular}{llll}
\hline $\begin{array}{l}\text { CFIR construct and } \\
\text { subconstruct }\end{array}$ & Clinicians' perceptions about CHWs & Representative quotes & $\begin{array}{l}\text { Influence of context on } \\
\text { implementation outcomes }\end{array}$
\end{tabular}

Outer setting: External policies and incentives

Inner setting: Implementation climate

Inner setting: Networks and communications

Inner setting: Networks and communication
Clinicians recognized that their time constraints in clinical care and lower priority accorded to prevention made CHWs advantageous over clinic-based counseling. But they worried that uncontrolled diabetes was a significant problem among many patients, and directing CHW resources towards prediabetes seemed to run contrary to an expectation (internal and external) that treatment be a priority over prevention. They felt that physicians who did not prioritize prediabetes might be reluctant to endorse a program focused on at-risk patients. Moreover, it took a long time to show outcomes from prevention, making it hard to change public perceptions about its importance. Clinicians perceived that CHWs could alleviate their time constraints for behavioral counseling. They showed an openness to absorbing this new intervention, consensus on its need, and receptivity to its potential organizational benefits. Clinicians acknowledged their own limitations in reaching patients - citing their comfort with CHWs being better at and more suited to culturally competent counseling than they themselves were-indicating an implementation climate where individuals were ready to complement their own skills and competencies with what CHWs could offer.

Reflecting on the logistics of contact and communication with CHWs, clinicians wanted to learn from $\mathrm{CHWs}$ and understand what CHWs observed about patients - they placed importance on dialogue with CHWs.

Clinicians were concerned about finding ways to structure an exchange without it burdening their already high workload and time constraints. They suggested regularly scheduled, consolidated feedback through written reports, inperson meetings, or notes in the EHR. Reports could focus on difficult patient cases or provide updates on patient activities and progress. CHWs could flag particular challenges or pose questions, say in an email. patient to do what's healthy," said a physician.

"Prediabetes is not a fatal disease, right? So it's a lot of energy being devoted to a preliminary thing that's not even quite a disease yet. That's where I think you may have problems getting providers to take this seriously. Prediabetes is ... not scary to providers. ... It's not dramatic..." said a physician.

"The problem with prediabetes and the population you are looking at is that it's hard to see an outcome ... the outcome is a little bit longer over time. ... One of the other things that we deal with in prevention all the time is, nobody thanks me for the heart attack they did not have," said a physician.

"The counseling I do is so minimal, in terms of what they really need," said a physician.

"The doctor has a few minutes to give [patients] some pointers about what to do, I have a few minutes, you know, but this way, someone can actually communicate with the patient," said a registered nurse.

"[CHWs] could be more specific about [diet and other counseling] than I could about what to eat," said a physician.

“... I'm comfortable with that ... with someone else being better at something [i.e., at culturally concordant counseling] than I am," said a physician.

One physician wanted to understand what CHWs observed about patients at their homes and about their cultural barriers or personal situation, including issues such as "domestic violence, ... food insecurity or housing insecurity..." as well as what the CHW was teaching the patient, so that he could reinforce that with the patient.

Any information the physician himself had access to, "particularly about patients' pre-diabetic condition, should be accessible to the community health worker, and then vice versa ... whatever documentation the CHW is using, I should have access to that as well." Getting updates from the CHW "would actually reinforce my utilization [of CHWs]. If I do not hear from them, then it'll be like it does not exist ...

[Getting updates] would motivate me," said a physician.

"I do not want to get inundated ... But maybe there could be a summation that I could read once a week, once a month .." said a physician.

One physician suggested that CHWs' reports could focus "on the patients where it's not going well. We almost do not have time to hear about the successes."

Another physician suggested focusing on changes achieved in patients' diet, exercise, and weight; patients' feelings about these changes; and CHWs' perceptions about opportunities to make future changes.
Clinicians felt that both public and professional expectations were to prioritize treatment over prevention of diabetes, which potentially diminished the perceived appropriateness and feasibility of using $\mathrm{CHWs}$ for at-risk patients.

Clinicians' openness to CHWs as a novel intervention and consensus on its need and potential benefits supported its perceived acceptability and appropriateness.

Clinicians' preference for bidirectional information exchange supported the prospective feasibility of their contact with CHWs.

Concerns about existing time constraints and prospective information overload from CHWs tempered the quantity and scope of informationsharing clinicians considered feasible in their practices. 
Table 4. (continued)

\begin{tabular}{llll}
\hline \hline $\begin{array}{l}\text { CFIR construct and } \\
\text { subconstruct }\end{array}$ & Clinicians' perceptions about CHWs & Representative quotes & $\begin{array}{l}\text { Influence of context on } \\
\text { implementation outcomes }\end{array}$
\end{tabular}

"So I would see a note [in the EHR] that would say ... met with patient to discuss prediabetes, we decided on the following interventions, increase exercise, change one serving of rice to quinoa or faro or something, then ... discussed with patient next month these goals were accomplished, these goals were not accomplished, overall patient feels good or bad or indifferent. That would be optimal I think," said a physician.

Inner setting: Structural characteristics
When reflecting on ways to incorporate CHWs into team structures and procedures, clinicians said it was essential that CHWs were familiar with and could help patients navigate the clinic's existing resources. They felt that the CHWs' priorities and workflow may not match that of the team, which could obviate CHWs' participation in team conferences. Clinicians said the $\mathrm{CHW}$ should interact widely with PC team members, especially registered nurses. Clinicians felt CHWs could enhance the team's overall functioning, such by helping registered nurses with panel management, but team tasks and workflow involving CHWs had to be developed and defined.
There could be times when "issues that are relevant to a $\mathrm{CHW}$ are not as important or relevant at the moment to the provider," which could lead to the CHWs' feeling as though they or their input was not included or valued, said one physician.

Registered nurses could be a bridge between CHWs and physicians. One nurse gave an example: "We have a social worker who is part of the [PC] team, and she and I often meet in the mornings and if there are pertinent patient issues ... we'll go through them, and we'll formulate a little plan. So, I like that style, it seems to work ... A lot of nurses feel really busy ... It would just depend on how much time the [CHW] would want to meet. That's the only problem I think."

In their present organizational procedures, "Nurse care managers [were] supposed to be our point people in the [PC] team model," but were delivering less sophisticated care than they potentially could, said a physician. A physician noted that, "In theory, [RNs] are supposed to do a little bit more of this panel management," but there was a gap in RNs' training and actual tasks. CHWs could step in and help RNs to manage the panel.

Workflow and division of responsibilities had to be designed. As one physician put it: "“... our diabetic nurses would be completely willing to get information about some of our patients [from CHWs]. But I do not anticipate them initiating some of the pull of information."
Clinicians' expectations about the potential for registered nurses to liaise with CHWs and for CHWs to improve team tasks such as panel management supported the feasibility of integrating CHWs into PC teams. with uncontrolled diabetes for whom I need a resource like that." One physician suggested that many medical professionals did not perceive prediabetes as an urgent priority and might be reluctant to endorse a program focused on at-risk patients. Another noted the challenge of shifting public perceptions about the need for preventive care. Physicians thus perceived it was difficult to change both providers' and patients' views about prevention, making it difficult to advocate for CHWs.

\section{Inner Setting}

The safety-net organizations' implementation climate, which includes its openness to learn and absorb change, positively influenced clinicians' perceptions about CHWs' appropriateness and feasibility. One physician said that having a $\mathrm{CHW}$ as part of the PC team "would motivate me more to screen, because now I have a partner, we can do something about it. We can actually affect change and have an impact." Clinicians noted that CHWs could alleviate their time constraints for behavioral counseling. "It's always going to come down to time ... how can [the CHW] help you with the limited amount of time you have to see these patients?" If a CHW accompanied a patient to a clinic visit, they could "reinforce the things [the physician] would say," to the patient outside of the visit." Clinicians clearly acknowledged their own limitations in reaching their patient population, saying, for example, that a $\mathrm{CHW}$ "would probably counsel about diet much 
better than I would," indicating their readiness to complement their own skills and competencies.

Clinicians' suggestions for ways to feasibly integrate CHWs in their practices highlighted the importance of networks and communications, meaning the nature of relationships and modes of information-sharing in the organization, and structural characteristics, which include the functional differentiation within an organization. For instance, when physicians reflected on logistics of contact and communication with CHWs, they were positive about the benefits of learning from CHWs. However, they were concerned about finding ways to structure an exchange "without it becoming invasive." They questioned whether having a CHW on their team would "slow [them] down," if it would "mean more phone calls" and an increased workload. Physicians worried about being presented with too much information, and suggested that regularly scheduled feedback, such as monthly or quarterly reports, or an email update, would suffice. One physician favored reading the CHWs' notes in the patient's chart before the patient's visit. They thus expressed varied and uncertain preferences for inperson communication with CHWs.

When reflecting on incorporating CHWs into team structures, clinicians emphasized it was essential that CHWs were knowledgeable not only about outside community-based resources, but also the clinic's existing internal resources, such as support from psychologists and nutritionists. Physicians emphasized the importance of CHWs interacting with the entire PC team to improve team functioning, but were unspecific about the position CHWs would formally occupy. Registered nurses (RNs) came up frequently as a preferred liaison between physicians and CHWs. A physician said that CHWs could "show up at some of the huddles" and communicate widely with team members, including the RN. A physician stated that RNs in her practice were not currently involved in prediabetes care, and CHWs could assist the RNs to review, for example, what patients were doing in terms of exercise and diet goals.

An RN manager reinforced the benefits of teamwork between RNs and CHWs. Since RNs "get to know [the] patients pretty well," they could share information with the CHW, such as the patient's history and tendencies, giving the CHW insights into individual patients and how to deal with them. Yet the question remained: how would procedures for that exchange be structured? One physician noted that the CHW's priorities and workflow may not match that of the team, which could obviate CHWs' participation in team conferences. Another noted that workflow procedures involving CHWs had to be developed and defined before challenges could be identified.

\section{DISCUSSION}

Three main themes emerge from our analysis of contextual factors underlying clinicians' perceptions about working with CHWs for diabetes prevention.
1. Clinicians were open to innovation and knew enough about CHWs to find them broadly acceptable for diabetes prevention, but had concerns about whether CHWs will fully benefit patients.

2. Clinicians perceived CHWs as appropriate for their practices and patients and for preventing diabetes, yet were concerned that diabetes treatment rather than prevention was the more urgent priority.

3. Clinicians' suggestions for ways to feasibly integrate CHWs into their practices underscored their concerns about the relative importance of prevention vs. treatment; the logistics, modes, and content of provider-CHW information exchange; and aligning CHWs' priorities, roles, and workflow with those of the rest of the PC team.

We elaborate on each theme below.

First, our results affirm other studies indicating clinicians' uncertainty about CHWs' training and knowledge. ${ }^{24}$ We understand this not as a reflection of clinicians' narrow or skeptical view, but rather partly as a product of their limited firsthand exposure to the technicalities of CHW models and to evaluative evidence from CHW programs. ${ }^{25}$ Clinicians' concerns about CHWs suggest the need to generate and, importantly, disseminate not only evidence about CHWs' effectiveness, but also operational details about CHW models, specifying how CHWs can be selected for their interpersonal skills and empathetic disposition towards patients' social needs, trained to respond to these needs, and integrated into PC teams. ${ }^{19,38,39}$ Clinicians in our study were open to CHWs as a welcome innovation for diabetes prevention and explicitly recognized the value of CHWs' cultural competence. The task ahead is to build on this organizational openness by widely informing clinicians about existing operational protocols and alternate possibilities for integrating CHWs as part of PC practice.

Second, our findings align with evidence suggesting that physicians do not uniformly consider screening for or treating prediabetes to be a high priority ${ }^{7}$ and may perceive counseling and treating prediabetes to be of uncertain effectiveness and a low-value use of clinical time and resources. ${ }^{40} \mathrm{CHWs}$ can help overcome clinicians' time barriers to offer lifestyle counseling and patients' inability to conform with healthful behavior change.

Our findings also emphasize the challenge physicians perceive in managing a panel of patients with multiple and competing disease conditions, such that prevention efforts may not gain their ready approval. Advocates of CHW programs have emphasized the need to invest in comprehensive, patient-centered programs. ${ }^{25,41}$ Such an approach would ease $\mathrm{CHW}$ integration into teams that care for diverse patients. Many studies of CHW programs have been conducted with disease-specific goals, ${ }^{42}$ partly as a function of the grant-based funding that has largely characterized CHW programs so far. As evidence of CHWs' cost-effectiveness has mounted ${ }^{43}$ and 
policy reforms have opened new financing sources and terms for $\mathrm{CHWs},{ }^{11,44} \mathrm{CHWs}$ have been hired directly by health care organizations. ${ }^{45} \mathrm{CHWs}$ are well placed to fulfill tasks that are broad-based, community-oriented, and not directly reimbursable, but that nonetheless contribute to improved health as part of a cost-effective and efficient model of care, ${ }^{45}$ one that is likely to alleviate physicians' concerns about using CHWs for disease-specific purposes. Concurrently, disseminating evidence that disease-specific $\mathrm{CHW}$ approaches have demonstrated effectiveness, such as for hypertension, ${ }^{46,47}$ diabetes, ${ }^{15,17}$, 22, 48, 49 and aspects of asthma management, ${ }^{50}$ is also important to alleviate physicians' concerns about CHWs.

Third, our findings underscore that introducing CHWs into PC practices entails recalibrating teamwork and workflow. Clinicians' diverse suggestions indicate the need to account for internal social networks, communication modes, and structural characteristics at both organizational and team levels. CHWs' duties within PC teams and their supervision and working relationships with $\mathrm{PC}$ team members can vary widely. $^{20,}{ }^{23}$ Leveraging CHWs' competencies and community knowledge will entail clarifying scope of practice and roles for all team members, including social workers, nurses, case managers, and other staff. ${ }^{23,48,51}$ While studies document this variation in CHW models, there is relatively less guidance on how to achieve CHW integration. Researchers have emphasized the need for operational protocols to guide CHW integration into PC teams. ${ }^{25-27}$ Implications also follow for CHW training and competencies, particularly training CHWs to navigate both clinical and community settings and to articulate their unique, distinct capabilities and purview of work within each setting. ${ }^{19,45}$

Studies additionally suggest that $\mathrm{CHW}$ integration will depend on clinic leaders who can champion the CHW model and its sustainability and a clinic culture that is open to innovation and prioritizes patients' non-medical needs. ${ }^{24} \mathrm{Be}-$ yond features of the PC team, the health care organization's commitment to the CHW model can contribute to successful integration of CHWs within PC teams. ${ }^{39}$ Clinicians in our study evinced a concern for the social determinants of their patients' health, and were open to CHWs as an innovation over available preventive care options. The views of organizational managers or administrators will be assessed in the next stage of this study, where we revisit clinicians' perspectives on CHWs 1 year into the intervention.

One limitation of our study is that, as noted above, it did not include the view of administrative stakeholders, whose support is important for the feasibility of strategies to integrate CHWs in PC teams, and neither did we interview other PC team members or CHWs. We plan to broaden the range of interviewees for the next phase of this study. Another limitation is that we did not directly observe clinic workflow, which would have provided additional contextual detail about the organizational setting. However, clinicians' views about workload as reported here are common to $\mathrm{PC}$ settings, 5,52 and were validated by two study team members who are also physicians at one of the study sites and one team member who has previously conducted studies at the other site.

\section{CONCLUSION}

These findings illuminate the influence of factors at multiple levels - the CHW model, features of the PC practice setting, features of the patient population in safety-net organizations - upon implementation outcomes. The successful delivery of CHW-led models of diabetes prevention will depend not only on CHW-patient interactions in community settings, but also on physicians', PC teams', and CHWs' shared understandings and interactions in clinical settings.

Acknowledgments: We are grateful to all clinicians at Bellevue Hospital and the Manhattan VA for sharing their perspectives with us. We thank Yixin Fang for advice and assistance on statistical analysis of survey data and Thomas Gepts and Shana Johnson for contributing to data synthesis and reviewing the manuscript.

Corresponding Author: Radhika Gore, PhD; NYU School of Medicine, New York, NY 10016, USA (e-mail: radhika.gore@nyulangone. org).

Funding Information This study has undergone peer-review by and is supported by the National Institutes of Health - National Institute of Diabetes and Digestive and Kidney Diseases under award number R18DK110740.

\section{Compliance with Ethical Standards:}

This study received all necessary ethics approvals from the NYU School of Medicine Institutional Review Board, the VA New York Harbor Healthcare System Office of Research \& Development Institutional Review Board, and the Bellevue Research Review Committee and New York City Health + Hospital's Research Administration.

Conflict of Interest: The authors declare that they do not have a conflict of interest.

Disclaimer: The content is solely the responsibility of the authors and does not necessarily represent the official views of the National Institutes of Health. The funding agency has not influenced the design of this study or the writing of this manuscript and will not influence the study's implementation.

\section{REFERENCES}

1. CDC. National Diabetes Statistics Report, 2017. Atlanta, GA: Centers for Disease Control and Prevention, US Department of Health and Human Services; 2017

2. Knowler WC, Fowler SE, Hamman RF, et al. 10-year follow-up of diabetes incidence and weight loss in the Diabetes Prevention Program Outcomes Study. Lancet 2009;374:1677-86.

3. Weber MB, Twombly JG, Venkat Narayan KM, Phillips LS. Lifestyle Interventions and the Prevention and Treatment of Type 2 Diabetes Am J Lifestyle Med 2010;4:468-80.

4. Li G, Zhang $\mathbf{P}$, Wang $\mathbf{J}$, et al. The long-term effect of lifestyle interventions to prevent diabetes in the China Da Qing Diabetes Prevention Study: a 20-year follow-up study. Lancet 2008;371:1783-9.

5. Yarnall KSH, Pollak KI, Østbye T, Krause KM, Michener JL. Primary Care: Is There Enough Time for Prevention? Am J Public Health 2003;93:635-41. 
6. Tseng E, Greer RC, O'Rourke P, et al. Survey of primary care providers' knowledge of screening for, diagnosing and managing prediabetes. J Gen Intern Med 2017;32:1172-8.

7. Mainous AG, Tanner RJ, Scuderi CB, Porter M, Carek PJ. Prediabetes Screening and Treatment in Diabetes Prevention: The Impact of Physician Attitudes. J Am Board Family Med 2016;29:663-71

8. Tang TS, Ayala GX, Cherrington A, Rana G. A Review of VolunteerBased Peer Support Interventions in Diabetes. Diabetes Spectrum 2011;24:85-98.

9. Dale JR, Williams SM, Bowyer V. What is the effect of peer support on diabetes outcomes in adults? A systematic review. Diabet Med 2012;29:1361-77.

10. Chang A, Patberg E, Cueto V, et al. Community Health Workers, Access to Care, and Service Utilization Among Florida Latinos: A Randomized Controlled Trial. Am J Public Health 2018;108:1249-51.

11. Islam N, Nadkarni SK, Zahn D, Skillman M, Kwon S, Trinh-Shevrin C. Integrating community health workers within Patient Protection and Affordable Care Act implementation. J Public Health Manag Pract 2015;21:42-50.

12. Gallant MP. The influence of social support on chronic illness selfmanagement: a review and directions for research. Health Educ Behav 2003;30:170-95.

13. Heisler M, Vijan S, Makki F, Piette JD. Diabetes control with reciprocal peer support versus nurse care management: a randomized trial. Ann Intern Med 2010;153:507-15.

14. Allicock M, Haynes-Maslow L, Carr C, et al. Training veterans to provide peer support in a weight-management program: MOVE! Prev Chronic Dis 2013; 10:E185.

15. Kim KB, Kim MT, Lee HB, Nguyen T, Bone LR, Levine D. Community Health Workers Versus Nurses as Counselors or Case Managers in a Self-Help Diabetes Management Program. Am J Public Health 2016;106:1052-8.

16. Spencer MS, Rosland A-M, Kieffer EC, et al. Effectiveness of a Community Health Worker Intervention Among African American and Latino Adults With Type 2 Diabetes: A Randomized Controlled Trial. Am J Public Health 2011;101:2253-60

17. Spencer MS, Kieffer EC, Sinco B, et al. Outcomes at 18 Months From a Community Health Worker and Peer Leader Diabetes Self-Management Program for Latino Adults. Diabetes Care 2018;41:1414-22.

18. American Diabetes Association. Prevention or Delay of Type 2 Diabetes: Standards of Medical Care in Diabetes-2018. Diabetes Care 2018;41(Supplement 1):S51-S4.

19. Islam N, Nadkarni S, Peretz $\mathbf{P}$, et al. Integration of Community Health Workers into Primary Care Health Systems: The Time for New York is NOW! New York, NY: NYU-CUNY Prevention Research Center; 2016.

20. Kim K, Choi J, Choi E, et al. Effects of Community-Based Health Worker Interventions to Improve Chronic Disease Management and Care Among Vulnerable Populations: A Systematic Review. Am J Public Health 2016;106

21. Kangovi S, Mitra N, Norton L, et al. Effect of Community Health Worker Support on Clinical Outcomes of Low-Income Patients Across Primary Care Facilities: A Randomized Clinical Trial. JAMA Intern Med 2018;178: 1635-43.

22. Long JA, Jahnle EC, Richardson DM, Loewenstein G, Volpp KG. Peer Mentoring and Financial Incentives to Improve Glucose Control in African American Veterans: A Randomized Trial. Ann Intern Med 2012;156:416-24.

23. Norris SL, Chowdhury FM, Le KV, et al. Effectiveness of community health workers in the care of persons with diabetes. Diabet Med 2006;23:544-56.

24. Rogers E, Manser S, Cleary J, Joseph A, Harwood E, Call K. Integrating Community Health Workers Into Medical Homes. Ann Fam Med 2018; 16:14-20.

25. Kangovi S, Grande D, Trinh-Shevrin C. From Rhetoric to Reality Community Health Workers in Post-Reform U.S. Health Care. N Engl J Med 2015;372:2277-9.

26. Wennerstrom A, Bui T, Harden-Barrios J, Price-Haywood EG. Integrating Community Health Workers Into a Patient-Centered Medical Home to Support Disease Self-Management Among Vietnamese Americans:Lessons Learned. Health Promot Pract 2015;16:72-83.

27. Bovbjerg RR, Eyster L, Ormond BA, Anderson T, Richardson E Integrating Community Health Workers into a Reformed Health Care System. Washington DC: The Urban Institute; 2013.

28. Johnson SL, Gunn VL. Community health workers as a component of the health care team. Pediatr Clin N Am 2015;62:1313-28.

29. Chinman M, Woodward EN, Curran GM, Hausmann LRM. Harnessing Implementation Science to Increase the Impact of Health Equity Research. Med Care 2017;55:S16-S23.

30. Islam N, Gepts T, Lief I, et al. Protocol for the CHORD project (community health outreach to reduce diabetes): a cluster-randomized community health worker trial to prevent diabetes. BMC Public Health 2018;18.
31. Levy N, Moynihan V, Nilo A, et al. The Mobile Insulin Titration Intervention (MITI) for Insulin Glargine Titration in an Urban, LowIncome Population: Randomized Controlled Trial Protocol. JMIR Res Protoc 2015;4:e31

32. Miller DR, Safford MM, Pogach LM. Who Has Diabetes? Best Estimates of Diabetes Prevalence in the Department of Veterans Affairs Based on Computerized Patient Data. Diabetes Care 2004;27:b10-b21.

33. Yin RK. Case study research: design and methods. 4th ed. Thousand Oaks, CA: Sage Publications, Inc.; 2009.

34. Cresswell JW, Poth CN. Qualitative Inquiry and Research Design: Choosing Among Five Approaches. Thousand Oaks, CA: Sage Publications, Inc.; 2018.

35. Tomoaia-Cotisel A, Scammon DL, Waitzman NJ, et al. Context matters: The experience of 14 research teams in systematically reporting contextual factors important for practice change. Ann Fam Med 2013;11:S115-S23.

36. Damschroder LJ, Aron DC, Keith RE, Kirsh SR, Alexander JA, Lowery JC. Fostering implementation of health services research findings into practice: a consolidated framework for advancing implementation science. Implement Sci 2009;4:50.

37. Proctor E, Silmere H, Raghavan $\mathbf{R}$, et al. Outcomes for implementation research: conceptual distinctions, measurement challenges, and research agenda. Admin Pol Ment Health 2011;38:65-76.

38. Perez M, Findley S, Mejia M, Martinez J. The Impact of Community Health Worker Training and Programs in NYC. J Health Care Poor Underserved 2006; 17:26-43.

39. Findley S, Matos S, Hicks A, Chang J, Reich D. Community health worker integration into the health care team accomplishes the triple aim in a patient-centered medical home: a Bronx tale. J Ambulatory Care Manag 2014;37:82-91.

40. Kandula NR, Moran MR, Tang JW, O'Brien MJ. Preventing Diabetes in Primary Care: Providers' Perspectives About Diagnosing and Treating Prediabetes. Clin Diabetes 2018;36:59-60.

41. SoRelle R, Shea JA, Shasha B, Keddem S, Long JA, Werner RM. Patient Engagement, Access to Care, and Perceptions of Competing Priorities in the VA Primary Care Setting. J Gen Intern Med 2019

42. CDC. Addressing Chronic Disease through Community Health Workers: A Policy and Systems-Level Approach. Atlanta, GA: National Center for Chronic Disease Prevention and Health Promotion, Division for Heart Disease and Stroke Prevention; 2015.

43. Landers S, Levinson M. Mounting Evidence of the Effectiveness and Versatility of Community Health Workers. Am J Public Health 2016;106:591-2.

44. Katzen A, Morgan M. Affordable Care Act Opportunities for Community Health Workers: How Medicaid Preventive Services, Medicaid Health Homes, and State Innovation Models are Including Community Health Workers. Cambridge, MA: Harvard Center for Health Law and Policy Innovation and PATHS [Providing Access to Healthy Solutions]; 2014.

45. Malcarney M-B, Pittman P, Quigley L, Horton $\mathbf{K}$, Seiler N. The Changing Roles of Community Health Workers. HSR: Health Services Res 2017;52:360-82.

46. Brownstein JN, Chowdhury FM, Norris SL, et al. Effectiveness of Community Health Workers in the Care of People with Hypertension. Am J Prev Med 2007;32:435-47.

47. Ursua RA, Aguilar DE, Wyatt LC, et al. A community health worker intervention to improve blood pressure among Filipino Americans with hypertension: A randomized controlled trial. Prev Med Rep 2018;11:42-8.

48. Collinsworth A. Vulimiri M. Snead C, Walton J. Community health workers in primary care practice: redesigning health care delivery systems to extend and improve diabetes care in underserved populations. Health Promot Pract 2014;15:51S-61S.

49. Kangovi S, Mitra N, Grande D, Huo H, Smith RA, Long JA. Community Health Worker Support for Disadvantaged Patients With Multiple Chronic Diseases: A Randomized Clinical Trial. Am J Public Health 2017:107:1660-7.

50. Parekh TM, Copeland CR, Dransfield MT, Cherrington A. Application of the community health worker model in adult asthma and COPD in the U.S.: a systematic review. BMC Pulmonary Med 2019;19:116.

51. Allen CG, Escoffery C, Satsangi A, Brownstein JN. Strategies to Improve the Integration of Community Health Workers Into Health Care Teams: "A Little Fish in a Big Pond". Prev Chronic Dis 2015;12.

52. Arndt B, Tuan W-J, White J, Schumacher J. Panel Workload Assessment in US Primary Care: Accounting for Non-Face-to-Face Panel Management Activities. J Am Board Fam Med 2014:27:530-7.

Publisher's Note Springer Nature remains neutral with regard to jurisdictional claims in published maps and institutional affiliations. 


\section{ANNEXURE. INTERVIEW GUIDE}

Topic

Panel management for prediabetes

Usual practices in diabetes prevention practices

Beliefs about CHW' role

Expectations and concerns about working with

CHWs for diabetes prevention
Questions

Generally, how do you and your team monitor your assigned panel of patients?

- How do you feel about the frequency with which you review outcomes for your panel?

How do you individually manage your patients with prediabetes?

- What obstacles do you face in providing care to your patients at risk for diabetes?

- What do you think are your patients' major barriers to diabetes prevention?

- What clinic-based and community-based resources do you offer patients?

How do you work with your team to manage your patients with prediabetes?

- What team members are involved in the care for patients with prediabetes?

- How are responsibilities for the care of patients with prediabetes delegated/divided among team members?

- How do you communicate with other team members regarding your patients' prediabetes care?

What is your current understanding of what a CHW is/does?

If you had access to a CHW, how could he/she help you (and your team) better manage your patients at-risk for diabetes?

- How do you envision the CHW interacting with you and your team?

- Would you want to receive updates from the CHW on patient progress? How and how often? Including what information?

What concerns do you have about working with a CHW? How could these concerns be addressed?

Are there any other comments, concerns, or suggestions about peer health coaching, integration of a CHW into your team, diabetes prevention, or this study that you would like to share? 